\title{
Human(ized) monoclonal antibodies in atopic patients - state of the art
}

\author{
ARZU DIDEM YALCIN ${ }^{1,2}$, KEVSER ONBASI 3 , RUSEN UZUN ${ }^{4}$, FELIX HERTH ${ }^{5}$, \\ PHILIPP ALBERT SCHNABEL
}

${ }^{1}$ Department of Internal Medicine, Allergy and Clinical Immunology, Genomics Research Center, Academia Sinica, Taipei, Taiwan ${ }^{2}$ Department of Allergy and Clinical Immunology, Antalya Training and Research Hospital, University of Medical Science, Antalya, Turkey ${ }^{3}$ Department of Internal Medicine, Allergy and Clinical Immunology, Dumlupınar University, Kütahya, Turkey

${ }^{4}$ Department of Pulmonology, Antalya Training and Research Hospital, University of Medical Science, Antalya, Turkey

${ }^{5}$ Department of Pulmonary and Critical Care Medicine, Thoraxklinik Translational Lung Research Center, University of Heidelberg,

Heidelberg, Germany

${ }^{6}$ Department of Pathology, University of Heidelberg, Heidelberg, Germany

\begin{abstract}
Asthma is an important chronic disease affecting a lot of people worldwide. Treatment options for asthma like biological agents are being developed more frequently nowadays. Despite a lot of treatment options, some patients still remain symptomatic. As more and more practitioners choose treatment with biologic agents as a convenient way of therapy, biologic agents and other valuable methods must be discovered in order to cope with a growing number of treatment agents. This manuscript emphasizes on new generation monoclonal human(ized) antibodies in asthmatics and off-label use. The first developed biologic agent is the anti-immunoglobulin E monoclonal antibody called omalizumab. Currently it is an approved treatment option for asthma.
\end{abstract}

Key words: asthma, omalizumab, anti IL-5, humanized monoclonal antibodies.

(Cent Eur J Immunol 2020; 45 (2): 195-201)

\section{Introduction}

Asthma is an important chronic disease affecting a lot of people worldwide [1]. Treatment options for asthma like biological agents are being developed more frequently nowadays. Despite a lot of treatment options, some patients still remain symptomatic. As more and more practitioners choose treatment with biologic agents as a convenient way of therapy, biologic agents and other valuable methods must be discovered in order to cope with a growing number of treatment agents. This special issue emphasizes on new generation monoclonal human(ized) antibodies in asthmatics [2-4].

The pathophysiological mechanisms underlying asthma, which is a heterogeneous disease, are characterized by interactive responses among various cell types and the hematopoietic cells of the adaptive and innate immune systems. Frequently is conventional therapy like inhaled steroids and beta-agonists sufficient for asthma symptoms. However, a little minority of the asthmatics is not controlled with conventional therapy. Therefore are new treatment options essential for severe asthmatic patients [5].

\section{Anti-IL-5 molecules}

Interleukins (IL) derived from T-helper-2 (Th2) cells and innate lymphoid cells play an important role in the pathogenesis of asthma. Monoclonal antibodies targeting these cytokines as treatment for severe asthma are expected to be beneficial [6]. Eosinophilic inflammation is an important event in the pathogenesis of asthma. IL-5 is a key cytokine that arranges eosinophil production, survival, maturation and recruitment of eosinophils to the inflammation [7]. Mepolizumab, reslizumab, and benralizumab are new developed monoclonal antibodies that target the cytokine IL-5. Mepolizumab and reslizumab have been approved by the US Food and Drug Administration (FDA) for the treatment of patients with severe asthma with an eosinophilic phenotype $[8,9]$. Mepolizumab and reslizumab binds directly to IL-5 ligand. These molecules effectively decreased circulating and sputum eosinophil counts, but they failed to improve airway mucosal eosinophilia, acute exacerbation rates, lung function and symptom scores in several studies. These disappointing results may be affected from inappropriate selection of the patients. In order to overcome the probable mechanistic limitations of early

Correspondence: Arzu Didem Yalcin, Department of Internal Medicine, Allergy and Clinical Immunology, Genomics Research Center, Academia Sinica, 11529 Tapei,Taiwan, e-mail: adidyal@yahoo.com Submitted: 16.03.2017; Accept ed: 13.04.2017 
anti-IL-5 agents, an anti-IL-5R monoclonal antibody was developed and called as benralizumab. Benralizumab, previously known as MEDI-563, is a humanized recombinant IgG1-k isotype monoclonal antibody. It was constructed from the mouse anti-human IL-5Ra mAbs generated by mice immunized with recombinant human IL-5Ra [10]. Clinical studies revealed that anti-IL-5 monoclonal therapies for asthma could be safe for slightly improving FEV1 (or FEV1\% of predicted value), quality of life, and reducing exacerbations risk and blood and sputum eosinophils. However these drugs have no significant effect on PEF, and SABA rescue use. These may be a result of patient selection. Further trials are required to clarify the optimal antibody for different patients [7].

\section{Anti-IL-4/IL-13 molecules}

Another investigated cytokine important in the inflammatory pathways in the pathogenesis of asthma is anti-IL-4. IL-4 is a pleiotropic cytokine secreted mainly by activated T cells. Mast cells, basophils, and eosinophils can also secret IL-4 [10, 11].

IL-4 is important in inducing IgE isotype switching, T cell polarization into $\mathrm{Th} 2$ cells, and generation of IL-4, IL-5, and IL-13 by Th2 cells. IL-4Ra is expressed on CD4+ and CD8+ T cells, B cells, macrophages, lung epithelial cells, airway goblet cells, and smooth muscle cells [11]. There is a functional homogeniety between IL-4 and IL-13. IL-4 can activate a heterodimeric receptor complex consisting of the IL-4 receptor a-subunit (IL-4Ra) and a C subunit. IL-13 can activate the IL-4Ra and the IL-13 receptor a1-subunit (IL-13Ra1) [5]. Both IL-4 and IL-13 can bind to heterodimeric combination of the a-subunit of the IL-13 receptor and the a-subunit of the IL-4 receptor. And this leads to signaling of both IL-4 and IL-13. Therefore will blocking IL-4R a with an antibody of this receptor chain expected to block the effects of both IL-4 and IL-13 [6].

Pascolizumab and VAK694 are anti-IL-4 neutralizing monoclonal antibodies. Also IL-4 receptor antagonist drugs like dupilumab, pitrakinra and AMG-317 have been discovered. Even a recombinant IL-4Ra that captures soluble IL-4 and prevents their binding to IL-4 receptors, has been developed. It is called altrakincept. However, further research on this drug was discontinued by its manufacturer, since the phase 3 clinical trial failed to confirm its earlier promising results. Since there is a high redundancy of IL-4 and IL-13 signaling, blocking of both IL-4 and IL-13 has been expected to be more efficient [11]. Dupilumab is a drug that inhibits signaling from IL-4 and IL-13 concomitantly. It is a molecule that binds to the alpha subunit of the IL-4 receptor. Phase II trials for dupilumab showed that asthma exacerbations were decreased in patients using this drug $[12,13]$. Dupilumab also improved lung function. It reduced the inhaled corticosteroid dose in the patient group. There was also an associated reduction in fractional exhaled nitric oxide with reduced serum concentrations of Th2-associated inflammatory markers such as CCL17 (TARC), CCL26 (eotaxin-3), and IgE [6]. These results are promising and further clinical trials will us show us the long-term efficacy of dupilumab [11].

Another similar drug that targets IL-4 is pitrakinra. It is a recombinant human IL-4 variant that competitively inhibits IL-4Ra to interfere with the actions of both IL-4 and IL-13. Studies on this competitive antagonist called pitrakinra revealed that it leads to significant reduction in asthma exacerbations and improves asthma symptoms in patients with eosinophilia [14]. Pitrakinra also attenuated the late-phase asthmatic response to allergen challenge in patients with mild atopic asthma [6]. The other drug called AMG317 was evaluated in another phase II trial in approximately 300 patients with moderate to severe asthma. Weekly injections over 12 weeks were well tolerated but did not have significant effects on the Asthma Control Questionnaire score (ACQ score; the primary outcome) [14].

IL-13 shares $30 \%$ homology with IL-4. IL-13 is secreted by Th2 cells, ILC2s, mast cells, basophils, and eosinophils. IL-13 has the potential to increase goblet-cell differentiation, and activation of fibroblasts. IL-13 production can induce an increase in bronchial hyperresponsiveness, and switching of B-cell antibody production towards IgE [6]. IL-13 is similar to IL-4 and uses the same signaling pathways. The high-affinity receptor of IL-13 is a heterodimer of IL-4Ra/IL-13Ra1. IL-13Ra1 is present on eosinophils, B cells, monocytes, macrophages, smooth muscle cells, lung epithelial cells, airway goblet cells, and endothelial cells. Biologicals that target IL-13 are anti-IL-13 mAbs: anrukinzumab, dectrekumab, GSK679586, IMA-026, lebrikizumab, RPC-4046, and tralokinumab [11].

Patients with severe asthma often have elevated levels in sputum despite therapy with high dose inhaled or oral corticosteroids. Lebrikizumab is one of the many humanized monoclonal antibodies that have been developed to specifically bind to IL-13 and inhibit its function [14]. In a randomized, double-blind, placebo-controlled study were 219 asthma patients observed and evaluated whether the drug lebrikizumab could alter the course of asthma. Lebrikizumab treatment was associated with improved lung function. Patients with high pretreatment levels of serum periostin had greater improvement in lung function with lebrikizumab. This therapy needs futher evaluation before being utilized in clinics [15]. Another monoclonal antibody called tralokinumab, an investigational human IL-13-neutralising immunoglobulin G4 monoclonal antibody, has been evaluated in adults with moderate to severe uncontrolled asthma despite controller therapies. Patients were randomly assigned to receive tralokinumab or placebo subcutaneously every 2 weeks for 13 weeks. Although 
it had an acceptable safety and tolerability, it did not reduce asthma exacerbations [16].

\section{Antithymic stromal lymphopoietin}

Thymic stromal lymphopoietin (TSLP) is an epithelial cell derived cytokine that may trigger allergic inflammation and, thus, play a role in allergic asthma [17]. It is an epithelial-derived cytokine and makes its effect through its receptor, TSLP-R, which is a heterodimeric receptor that consists of the IL-7 receptor alpha chain (IL-7Ra) and the TSLP receptor alpha chain 1 (TSLPRa). In hematopoietic cells, TSLP-R is mainly expressed in DCs, monocytes, B cells, T cells, NK cells, invariant natural killer T (iNKT) cells, eosinophils, basophils, and mast cells [11].

A human anti TSLP monoclonal immunoglobulin G2 lambda (AMG 157) that binds human TSLP and prevents receptor interaction was assessed in a trial. Randomly assigned 31 patients with mild allergic asthma received AMG 157 (700 mg) or placebo intravenously, once a month for three doses. The primary outcome, the maximum percentage decrease in the FEV during the late asthmatic response was 45.9 percent less in the AMG 157 group than the placebo group on day 84. AMG 157 reduced allergen induced bronchoconstriction and airway inflammation. No serious adverse effects were reported. Further studies on this drug are planned to clarify its use in clinical practice [17].

\section{Anti-IL-9 monoclonal antibody}

IL-9 is a Th2 cytokine and a T cell and mast cell growth factor. Anti-IL-9 antibody-treatment has been shown to protect from allergen-induced airway remodeling, with a concomitant reduction in mature mast cell numbers and activation. It can also decrease expression of the profibrotic mediators transforming growth factor TGF)-b1, vascular endothelial growth factor (EGF), and fibroblast growth factor-2 (FGF-2) in the lung. The function of IL-9 in allergy has been investigated for its pleiotropic activities on cell types associated with allergic diseases including Th2 lymphocytes, mast cells, B cells, eosinophils, and airway epithelial cells. An anti-IL-9 monoclonal antibody (MEDI-528) has been studied in a clinical trial on 327 asthmatic subjects. Patients were randomized to receive placebo or one of three doses of MEDI-528 (dosage 30,100 , or $300 \mathrm{mg}$ s.c. twice weekly for 4 weeks) in addition to their usual asthma medications. The addition of MEDI-528 to existing asthma controller medications did not improve ACQ-6 scores, asthma exacerbation rates, or FEV1 values. Further clinical trials are needed to explore this drug for altering the course of asthma. Thus, the potential clinical benefit of targeting IL-9 or its receptor in the treatment of asthma remains to be shown in further studies [5].

\section{Anti-IL-2 antibody}

Allergen exposure can stimulate IL-2 and its receptor expression (IL -2R) a chain (sCD25) in airways of patients with severe asthma. Daclizumab is a humanized monoclonal antibody that binds specifically to the a subunit (CD25) of the high-affinity IL-2R, and inhibits IL-2 binding and its biological activity. Daclizumab can inhibit various $\mathrm{T}$ cell functions, including $\mathrm{T}$ cell proliferation and cytokine production. It has been investigated in a randomized controlled study. The drug has the potential to improve pulmonary function and asthma control in patients moderate to severe chronic asthma [18]. The risk of immunosuppression in clinical practice needs to be clarified.

\section{Anti-GATA3-spesific DNAzyme}

Approximately half of the asthmatic patients exhibit a Th2 type in response to allergen exposure. This Th2 endotype is characterized by a predominant activation of Th2 cells that produce cytokines such as IL-4, IL-5, and IL-13. The expression and production of all these Th2 cytokines have been shown to be controlled by the zinc finger transcription factor GATA3, which is essential for Th2-cell differentiation and activation. It is considered to be the master transcription factor of the Th2 pathway of immune activation. Therefore could be interventions to disrupt this immune network, a synthetic DNA molecule (DNAzyme), that binds to GATA3 messenger RNA and cleaves it, a solution. This synthetic molecule called SB010 could significantly attenuate both late and early asthmatic responses after allergen provocation in patients with allergic asthma. Biomarker analysis after this drug showed an attenuation of Th2-regulated inflammatory responses [19].

\section{Anti-IL-17 antibody}

Although half of the asthma patients exhibit a Th2 type endotype, some remaining patients exhibit a Th17 driven endotype. This subpopulation is characterized with a Th17 driven inflammation. Th17 cells can contribute to airway hyperresponsiveness by recruiting both eosinophils and neutrophils. Therefore has ben IL-17 receptor blocking suggested to beneficial in asthma treatment [20].

Biologicals targeting IL-17 include an anti-IL-17A mAb: secukinumab and an anti-IL-17 receptor $\mathrm{mAb}$ : brodalumab. Although the inhibition of IL-17 receptor A had no effect on subjects with asthma as a whole, a subgroup analysis showed an effect with uncertain significance. Further studies are needed to determine the role of secukinumab in asthma [11]. Brodalumab (AMG 827) is a human, anti-IL-17RA immunoglobulin G2 (IgG2) monoclonal antibody that binds with high affinity to human IL-17RA, blocking the biologic activity of IL-17A, $-17 \mathrm{~F},-17 \mathrm{~A} / \mathrm{F}$ heterodimer, and -17E (IL-25). Broda- 
lumab can block IL-25 activity and IL-17A and IL-17F. In a randomized controlled study were 302 patients taking this drug evaluated and at the end of the study there was no evidence for an effect of brodalumab in these patients. Further studies may clarify the potential of this drug [20].

\section{Anti TNF antibodies}

In addition, human(ized) monoclonal antibodies (HMA) evaluated for the treatment of severe persistent asthma (SPA), but not approved after Phase II trial are as follows; Infliximab (recombinant human-murine chimeric anti-TNFa monoclonal antibody Infliximab), etanercept (soluble TNFa receptor fusion protein), and golimumab (fully human TNFa-blocking antibody) [5]. The expression of TNF alfa is increased in asthma in association with airway neutrophilia. Berry and colleagues have demonstrated that the TNF-a axis is upregulated in patients with refractory asthma, as evidenced by the increased expression of membrane-bound TNF-a, TNF receptor 1 , and TNF-a- converting enzyme by peripheral-blood monocytes [21]. Treatment with golimumab did not demonstrate a favorable risk-benefit profile in patients with severe persistent asthma [22]. A study with etanercept showed a small decrease in asthma exacerbations was observed in a randomized placebo controlled study [23]. In a case-series report was it told that in severe, uncontrolled, steroid-dependent asthma infliximab could reduce exacerbations and hospitalizations [24]. In some severe refractory asthma endotypes anti-TNFa therapy may have a role. However, it should be kept in mind that these agents have some safety concerns and should used carefully only in some severe refractory asthma endotypes [5].

\section{Anti-IgE}

The first developed biologic agent is the anti-immunoglobulin E monoclonal antibody called omalizumab. Currently it is an approved treatment option for asthma [5].

The other human(ized) promising monoclonal antibody drug developed, but not approved yet is ligeluzimab. Ligeluzimab binds with very high affinity to the $\mathrm{Ce} 3$ domain of IgE. Ligeluzumab may provide longer supression of IgE. Trials with this biologic agent are ongoing [5].

\section{Anti-IgE: off-label use}

\section{Non-atopic asthma}

The off-label use of omalizumab in patients with uncontrolled non-atopic asthma has resulted in a decrease in exacerbation rates and improvement in asthma symptom scores. In a study conducted in 2013, omalizumab was administered to 266 patients with severe allergic asthma and 29 patients with non-atopic severe asthma for two years, and the study found a decline in the exacerbation rate, in- crease in the quality of life, and significantly improved disease control in both groups [25-27]. In two studies which used omalizumab in a group of patients with non-atopic severe asthma, the authors observed downregulation of FcRI expression in the basophils and increased FEV1.

\section{Nasal polyposis, allergic rhinitis, and allergic bronchopulmonary aspergillosis}

Allergic bronchopulmonary aspergillosis affects 7 to $9 \%$ of patients with cystic fibrosis (CF) and 1 to $2 \%$ of patients with asthma, posing a diagnostic challenge [28, 29]. Cases series related to ABPA were first published in 2007. In a series of eight cases with cystic fibrosis and ABPA published by Tanou et al. [30] in 2014, the authors reported increased FEV1, improved respiratory symptoms, and reduced steroid consumption. In a series of six cases with CF diagnosed with ABPA published by Lehman et al. [31], the authors reported improved symptoms in patients receiving omalizumab, whereas the efficacy of the treatment was less pronounced in patients diagnosed with ABPA and long disease duration and in patients who developed progressive lung problems [31]. In a series of 14 patients with severe asthma and ABPA in 2015, Aydin et al. [32] showed that 11 patients achieved complete and three patients achieved partial response. The authors also reported an overall improvement in the pulmonary functions and respiratory symptoms with a statistically significant reduction in the use of oral corticosteroids (OCS) and number of disease episodes. Also, the patients with a total immunoglobulin $\mathrm{E}$ (IgE) level of $<1,000$ IU showed a better response to omalizumab, compared to those with a total IgE level of $>1,000$ IU. However, this finding was found to have a low-evidence level, considering the lack of large-scale, prospective case series and randomized and placebo-controlled studies.

Efficacy and safety of omalizumab were first evaluated in a randomized, double-blind, placebo-controlled study of 221 patients with seasonal allergic rhinitis in 2002 [33]. This study reported a significant symptomatic relief up to $48 \%$ in the combination treatment group (specific immunotherapy [SIT]+omalizumab), compared to SIT group alone. A randomized study in Japan reported a significant improvement in daily nasal and eye symptoms in patients with seasonal allergic rhinitis receiving omalizumab [34].

In 2007, a randomized placebo-controlled study of eight patients was the first to report reduced rates of postoperative polyp recurrence in patients with atopic asthma and nasal polyps (NP) [35]. In a study of 19 patients with severe asthma and NP in 2011, Vennera et al. [36] reported symptom reduction and disease stabilization with the use of omalizumab treatment. In addition, Tajiri et al. [37] evaluated omalizumab in patients with severe asthma and NP, and reported significant improvements in nasal symptoms, asthma control, and sinus tomography results. However, not all studies were able to show the beneficial 
effects of the treatment. In a randomized, double-blind, placebo-controlled study of patients with chronic rhinosinusitis receiving omalizumab, Pinto et al. [38] showed improvement in the Sino-Nasal Outcome Test (SNOT-20) scores at three, five, and six months, although there was no significant difference in the scores compared to the control group. The aforementioned study did not observe any changes in the quality of life, symptom scores, cellular inflammation, nasal passage, and olfactory test parameters.

\section{Atopic dermatitis and food allergy}

In a series of three patients published in 2005, the authors reported no response after four months of treatment [39], while Lane et al. [40] published a series of three patients in the same study period and reported successful treatment outcomes of severe AD using omalizumab [40]. In addition, a pilot study of 21 patients published in 2009 found a statistically significant clinical improvement in all patients [41]. Another series of three patients published in 2011 reported significant improvement in the Eczema Area and Severity Index (EASI) and itching severity score in patients with severe $\mathrm{AD}$ unresponsive to conventional treatment and those with elevated IgE levels [42]. A study of 11 patients published in the same year reported reduced SCORing Atopic Dermatitis (SCORAD) scores, reduced symptoms, and significant improvement in the quality of life (3). The efficacy of omalizumab was also evaluated in 20 adults with severe AD in a prospective, 28-week, open-label study conducted by Hotze et al. [43] in 2014. The authors reported no response to treatment in seven patients harboring filaggrin gene mutation (FLG), while there was a significant improvement in the remaining eight patients. The authors also concluded that the patients with FLG gene mutation were prone to achieve lower response to omalizumab.

Furthermore, in food-related immunotherapy (IT) studies, omalizumab initiated before or received simultaneously with the treatment facilitated the development of tolerance. In a series of 11 patients with cow milk allergy scheduled for IT, 10 patients tolerated daily intake of $8 \mathrm{~g}$ cow milk after the initiation of omalizumab, nine weeks before IT, and combination with IT treatment, thereafter [44]. Another use of this treatment is to facilitate a rapid and safe transition to the maintenance phase in patients with food allergy receiving oral IT [45]. Another use of the treatment in food allergy is eosinophillic esophagitis developing in association with multiple food allergies. Also, in a study administered omalizumab to patients with eosinophillic esophagitis, the authors found reduced allergic symptoms and improved quality of life, although there was no change in endoscopic and histologic characteristics of the disease [46].

\section{Food allergy and anaphylaxis}

Anaphylaxis can occur as a result of exposure to various allergens such as food, drug, and venom; however, no triggering factor can be shown in some cases, of which the latter is known as idiopathic anaphylaxis. The patients with elevated baseline tryptase levels or those diagnosed with mastocytosis are expected to have higher rates of anaphylaxis with a more aggressive course of disease. In particular, patients with venom allergy on IT may experience some difficulties in switching to maintenance dose. Severe anaphylactic episodes can be observed in patients diagnosed with mastocytosis on IT due to venom allergy. In addition, IT combined with omalizumab has enabled a safe transition to the maintenance phase in this group of patients [47-49]. Addition of omalizumab to rush and ultra-rush venom IT protocols has increased the success of IT and enabled a safer transition to the maintenance phase. Another use of omalizumab is to prevent recurrent anaphylactic episodes in patients who are unable to be controlled with conventional therapies [50-55]. There are case reports on the role of this treatment in preventing idiopathic anaphylactic episodes [56-58].

Several studies have reported asthma symptom control, improved quality of life, and development of tolerance to aspirin in two patients with the use of omalizumab in patients with aspirin-induced airway disease, nasal polyps, and severe asthma [59, 60]. Two patients with recurrent insulin allergy, despite the use of desensitization protocols, and one patient of carboplatin allergy during carboplatin therapy due to ovarian cancer successfully continued their treatment with the addition of omalizumab to the treatment [61-63].

\section{Conclusions}

In conclusion, although omalizumab has been approved for the treatment of severe allergic asthma and chronic idiopathic urticaria, it offers an off-label use as a final resort in many allergic diseases. Recent studies have shown that omalizumab is effective in treating bullous pemphigoid, Stevens-Johnson syndrome (SJS)/toxic epidermal necrolysis (TEN, Type-III/IV reaction, such as graft versus host disease), Netherton syndrome, asthma and chronic obstructive pulmonary disease overlap syndrome (ACOS). Its effects on soluble inflammatory markers, such as sCD200, sTRAIL, hematopoietic cells, Th1/2 cytokines (CXCL8; IL-1B; IL-4; IL-5; IL17A), total antioxidant capacity, hydrogen peroxide, malondialdehyde and total nitric oxide concentrations were demonstrated in several studies [64-70].

Monoclonal antibodies are a candidate for use in several indications with the contribution of large-scale studies to the literature in the near future.

\section{Acknowledgements}

The authors would like to thank Prof. Dr. Tse Wen Chang and Prof. Dr Arzu Mirici.

The authors declare no conflict of interest. 


\section{References}

1. Beasley R, Semprini A, Mitchell EA (2015): Risk factors for asthma: is prevention possible? Lancet 386: 1075-1085.

2. Yalcin AD, Celik B, Gumuslu S (2014): D-dimer levels decreased in severe allergic asthma and chronic urticaria patients with the omalizumab treatment Expert Opin Biol Ther 14: 283-286.

3. Yalcin AD (2015): Advances in anti-IgE therapy. Biomed Res Int 2015: 317465.

4. Yalcin AD, Cilli A, Bisgin A, et al. (2013): Omalizumab is effective in treating severe asthma in patients with severe cardiovascular complications and its effects on SCD200, d-dimer, CXCL8, 25-hydroxyvitamin D and IL-1ß levels. Expert Opin Biol Ther 13: 1335-1341.

5. Mitchell PD, El-Gammal AI, O’Byrne PM (2016): Emerging monoclonal antibodies as targeted innovative therapeutic approaches to asthma. Clin Pharmacol Ther 99: 38-48.

6. Chung KF (2015): Targeting the interleukin pathway in the treatment of asthma. Lancet 386: 1086-1096.

7. Wang FP, Liu T, Lan Z, et al. (2016): Efficacy and Safety of Anti-Interleukin-5 Therapy in Patients with Asthma: A Systematic Review and Meta-Analysis. PLoS One 11: e0166833.

8. Ortega HG, Liu MC, Pavord ID, et al. (2014): Mepolizumab treatment in patients with severe eosinophilic asthma. N Engl J Med 371: 1198-1207.

9. Castro M, Zangrilli J, Wechsler ME, et al. (2015): Reslizumab for inadequately controlled asthma with elevated blood eosinophil counts: results from two multicentre, parallel, double-blind, randomised, placebo-controlled, phase 3 trials. Lancet Respir Med 3: 355-366.

10. Khorasanizadeh M, Eskian M, Assa'ad AHKhorasanizadeh M, et al. (2016): Efficacy and Safety of Benralizumab, a Monoclonal Antibody against IL-5Ra, in Uncontrolled Eosinophilic Asthma. Int Rev Immunol 35: 294-311.

11. Tan HT, Sugita K, Akdis CA (2016): Novel Biologicals for the Treatment of Allergic Diseases and Asthma. Curr Allergy Asthma Rep 16: 70.

12. Wenzel S, Ford L, Pearlman D, et al. (2013): Dupilumab in persistent asthma with elevated eosinophil levels. N Engl J Med 368: 2455-2466.

13. Wenzel S, Castro M, Corren J, et al. (2016): Dupilumab efficacy and safety in adults with uncontrolled persistent asthma despite use of medium-to-high-dose inhaled corticosteroids plus a long-acting $\$ 2$ agonist: a randomised double-blind placebo-controlled pivotal phase $2 \mathrm{~b}$ dose-ranging trial. Lancet 388: 31-44.

14. Hambly N, Nair P (2014): Monoclonal antibodies for the treatment of refractory asthma. Curr Opin Pulm Med 20: 87-94.

15. Corren J, Lemanske RF, Hanania NA, et al. (2011): Lebrikizumab treatment in adults with asthma. N Engl J Med 365: 1088-1098.

16. Piper E, Brightling C, Niven R, et al. (2013): A phase II placebo-controlled study of tralokinumab in moderate-to-severe asthma. Eur Respir J 41: 330-338.

17. Gauvreau GM, O’Byrne PM, Boulet LP, et al. (2014): Effects of an anti-TSLP antibody on allergen-induced asthmatic responses. N Engl J Med 370: 2102-2110.

18. Powell C, Milan SJ, Dwan K, et al. (2015): Mepolizumab versus placebo for asthma. Cochrane Database Syst Rev 2015: CD010834.

19. Krug N, Hohlfeld JM, Kirsten AM, et al. (2015): Allergen-induced asthmatic responses modified by a GATA3-specific DNAzyme. N Engl J Med 372: 1987-1995.
20. Busse WW, Holgate S, Kerwin E, et al. (2013): Randomized, double-blind, placebo-controlled study of brodalumab, a human anti-IL-17 receptor monoclonal antibody, in moderate to severe asthma. Am J Respir Crit Care Med 188: 1294-1302.

21. Blume C, Reale R, Held M, et al. (2017): Cellular crosstalk between airway epithelial and endothelial cells regulates barrier functions during exposure to double-stranded RNA. Immun Inflamm Dis 5: 45-56.

22. Wenzel SE, Barnes PJ, Bleecker ER, et al. (2009): A randomized, double-blind, placebo-controlled study of tumor necrosis factor-alpha blockade in severe persistent asthma. Am J Respir Crit Care Med 179: 549-558.

23. Morjaria JB, Chauhan AJ, Babu KS, et al. (2008): The role of a soluble TNFalpha receptor fusion protein (etanercept) in corticosteroid refractory asthma: a double blind, randomised, placebo controlled trial. Thorax 63: 584-591.

24. Taille C, Poulet C, Marchand-Adam S, et al. (2013): Monoclonal Anti-TNF-a Antibodies for Severe Steroid-Dependent Asthma: A Case Series. Open Respir Med J 7: 21-25.

25. Domingo C, Pomares X, Angril N, et al. (2013):Effectiveness of omalizumab in non-allergic severe asthma. J Biol Regul Homeost Agents 27: 45-53.

26. Garcia G, Magnan A, Chiron R, et al. (2013): A proof-ofconcept, randomized, controlled trial of omalizumab in patients with severe, difficult-to-control, nonatopic asthma. Chest 144: 411-419.

27. de Llano LP, Vennera Mdel C, Alvarez FJ, et al. (2013): Effects of omalizumab in non-atopic asthma: results from a Spanish multicenter registry. J Asthma 50: 296-301.

28. Laufer P, Fink JN, Bruns WT et al. (1984): Allergic bronchopulmonary aspergillosis in cystic fibrosis. J Allergy Clin Immunol 73: 44-48.

29. Shah A, Panjabi C (2016): Allergic Bronchopulmonary Aspergillosis: A Perplexing Clinical Entity. Allergy Asthma Immunol Res 8: 282-297.

30. Tanou K, Zintzaras E, Kaditis AG (2014): Omalizumab therapy for allergic bronchopulmonary aspergillosis in children with cystic fibrosis: a synthesis of published evidence. Pediatr Pulmonol 49: 503-507.

31. Lehmann S, Pfannenstiel C, Friedrichs F, et al. (2014): Omalizumab: a new treatment option for allergic bronchopulmonary aspergillosis in patients with cystic fibrosis. Ther Adv Respir Dis 8: 141-149.

32. Aydin Ö, Sözener ZÇ, Soyyigit S, et al. (2015): Omalizumab in the treatment of allergic bronchopulmonary aspergillosis: One center's experience with 14 cases. Allergy Asthma Proc 36: 493-500.

33. Navinés-Ferrer A, Serrano-Candelas E, Molina-Molina GJ, Martín M (2016): IgE-Related Chronic Diseases and Anti-IgEBased Treatments. J Immunol Res 2016: 8163803.

34. Okubo K, Nagakura T (2008): Anti-IgE antibody therapy for Japanese cedar pollinosis: omalizumab update. Allergol Int 57: 205-209.

35. Hong CJ, Tsang AC, Quinn JG, et al. (2015): Anti-IgE monoclonal antibody therapy for the treatment of chronic rhinosinusitis: a systematic review. Syst Rev 18: 166.

36. Vennera Mdel C, Picado C, Mullol J, et al. (2011): Efficacy of omalizumab in the treatment of nasal polyps. Thorax 66: 824-825.

37. Tajiri T, Matsumoto H, Hiraumi H, et al. (2013): Efficacy of omalizumab in eosinophilic chronic rhinosinusitis patients with asthma. Ann Allergy Asthma Immunol 110: 387-388. 
38. Pinto JM, Mehta N, DiTineo M, et al. (2010): A randomized, double-blind, placebo-controlled trial of anti-IgE for chronic rhinosinusitis. Rhinology 48: 318-324.

39. Lee JH, Son SW, Cho SH (2016): A Comprehensive Review of the Treatment of Atopic Eczema. Allergy Asthma Immunol Res 8: 181-190.

40. Lane JE, Cheyney JM, Lane TN, et al. (2006): Treatment of recalcitrant atopic dermatitis with omalizumab. J Am Acad Dermatol 54: 68-72.

41. Sheinkopf LE, Rafi AW, Do LT, et al. (2008): Efficacy of omalizumab in the treatment of atopic dermatitis: a pilot study. Allergy Asthma Proc 29: 530-537.

42. Thaiwat S, Sangasapaviliya A (2011): Omalizumab treatment in severe adult atopic dermatitis. Asian Pac J Allergy Immunol 29: 357-360.

43. Hotze M, Baurecht H, Rodríguez E, et al. (2014):Increased efficacy of omalizumab in atopic dermatitis patients with wild-type filaggrin status and higher serum levels of phosphatidylcholines. Allergy 69: 132-135.

44. Nadeau KC, Schneider LC, Hoyte L, et al. (2011): Rapid oral desensitization in combination with omalizumab therapy in patients with cow's milk allergy. J Allergy Clin Immunol 127: 1622-1624.

45. Begin P, Dominguez T, Wilson SP, et al. (2014): Phase 1 results of safety and tolerability in a rush oral immunotherapy protocol to multiple foods using Omalizumab. Allergy Asthma Clin Immunol 10: 7.

46. Rocha R, Vitor AB, Trindade E, et al. (2011): Omalizumab in the treatment of eosinophilic esophagitis and food allergy. Eur J Pediatr 170: 1471-1474.

47. Kontou-Fili K (2008): High omalizumab dose controls recurrent reactions to venom immunotherapy in indolent systemic mastocytosis. Allergy 63: 376-378.

48. Sokol KC, Ghazi A, Kelly BC, et al. (2014): Omalizumab as a desensitizing agent and treatment in mastocytosis: a review of the literature and case report. J Allergy Clin Immunol Pract 2: $266-270$

49. da Silva EN, Randall KL (2013): Omalizumab mitigates anaphylaxis during ultrarush honey bee venom immunotherapy in monoclonal mast cell activation syndrome. J Allergy Clin Immunol Pract 1: 687-688.

50. Douglass JA, Carroll K, Voskamp A, et al. (2010): Omalizum$\mathrm{ab}$ is effective in treating systemic mastocytosis in a nonatopic patient. Allergy 65: 926-927.

51. Jagdis A, Vadas P (2014): Omalizumab effectively prevents recurrent refractory anaphylaxis in a patient with monoclonal mast cell activation syndrome. Ann Allergy Asthma Immunol 113: 115-116.

52. Paraskevopoulos G, Sifnaios E, Christodoulopoulos K, et al. (2013): Successful treatment of mastocytic anaphylactic episodes with reduction of skin mast cells after anti-IgE therapy. Eur Ann Allergy Clin Immunol 45: 52-55.

53. Matito A, Blazquez-Goni C, Morgado JM, et al. (2013): Shortterm omalizumab treatment in an adolescent with cutaneous mastocytosis. Ann Allergy Asthma Immunol 111: 425-426.

54. Bell MC, Jackson DJ (2012): Prevention of anaphylaxis related to mast cell activation syndrome with omalizumab. Ann Allergy Asthma Immunol 108: 383-384.

55. Kibsgaard L, Skjold T, Deleuran M, et al. (2014): Omalizumab induced remission of idiopathic anaphylaxis in a patient suffering from indolent systemic mastocytosis. Acta Derm Venereol 94: 363-364.
56. Lee J (2014):Successful prevention of recurrent anaphylactic events with anti-immunoglobulin E therapy. Asia Pac Allergy 4: 126-128.

57. Demirtürk M, Gelincik A, Colakoglu B, et al. (2012): Promising option in the prevention of idiopathic anaphylaxis: omalizumab. J Dermatol 39: 552-554.

58. Jones JD, Marney SR Jr, Fahrenholz JM (2008): Idiopathic anaphylaxis successfully treated with omalizumab. Ann Allergy Asthma Immunol 101: 550-551.

59. Bobolea I, Barranco P, Fiandor A, et al. (2010): Omalizumab: a potential new therapeutic approach for aspirin-exacerbated respiratory disease. J Investig Allergol Clin Immunol 20: 448-449.

60. Matheu V, Franco A, Perez E, et al. (2007): Omalizumab for drug allergy. J Allergy Clin Immunol 120: 1471-1472; author reply $1472-1473$.

61. Cavelti-Weder C, Muggli B, Keller C, et al. (2012): Successful use of omalizumab in an inadequately controlled type 2 diabetic patient with severe insulin allergy. Diabetes Care 35: e41.

62. Yong PF, Malik R, Arif S, et al. (2009): Rituximab and omalizumab in severe, refractory insulin allergy. N Engl J Med 360: 1045-1047.

63. Ojaimi S, Harnett PR, Fulcher DA (2014): Successful carboplatin desensitization by using omalizumab and paradoxical diminution of total IgE levels. J Allergy Clin Immunol Pract 2: $105-106$.

64. Uzun R, Yalcin AD, Celik B, et al. (2016): Levofloxacin Induced Toxic Epidermal Necrolysis: Successful Therapy with Omalizumab (Anti-IgE) and Pulse Prednisolone. Am J Case Rep 17: 666-671.

65. Yalcin AD (2016): A case of netherton syndrome: successful treatment with omalizumab and pulse prednisolone and its effects on cytokines and immunoglobulin levels. Immunopharmacol Immunotoxicol 38: 162-166.

66. Yalcin AD, Celik B, Yalcin AN (2016): Omalizumab (anti-IgE) therapy in the asthma-COPD overlap syndrome (ACOS) and its effects on circulating cytokine levels. Immunopharmacol Immunotoxicol 38: 253-256.

67. Yalcin AD, Genc GE, Celik B, et al. (2014): Anti-IgE monoclonal antibody (omalizumab) is effective in treating bullous pemphigoid and its effects on soluble CD200. Clin Lab 60: 523-524.

68. Yalcin AD, Bisgin A, Gorczynski RM (2012): IL-8, IL10, TGF- $\beta$ and GCSF levels were increased in severe persistent allergic asthma patients with the anti-IgE treatment. Mediators Inflammation 2012: 720976.

69. Yalcin AD, Bisgin A (2012): The Relation of sTRAIL Levels and Quality of Life In Omalizumab Using Severe Persistent Allergic Asthma Patients (WISC 2012: WAO Outstanding Abstract Award). Med Sci Monit 18: LE9-10.

70. Yalcin AD, Ucar S, Gumuslu S, Strauss L. Effects of Omalizumab on Eosinophil Cationic Peptid, 25-Hydroxyvitamin-D, IL-1 1 , and sCD200 in a cases of Samter's syndrome: 36 Months follow-up. Immunopharmacology And Immunotoxicology; doi: 10.3109/08923973.213.811598. 